\title{
Nanoscale Hyperspectral Characterization of Source Rock in Unconventional Reservoirs using Photo-Induced Force Microscopy
}

\author{
Shannon L. Eichmann ${ }^{1}$, Derek Nowak ${ }^{2}$, David Jacobi ${ }^{1}$, and Nancy A. Burnham ${ }^{3}$ \\ 1. Aramco Services Company: Aramco Research Center - Houston, Houston, USA. \\ 2. Molecular Vista, San Jose, USA. \\ 3. Worcester Polytechnic Institute, Physics \& Biomedical Engr. Departments, Worcester, USA.
}

In unconventional reservoirs, a source rock is a conglomeration of inorganic minerals (i.e. silicates and carbonates) interspersed with finely distributed organic matter, consisting mainly of kerogen [1]. Atomic Force Microscopy (AFM) is a powerful technique used to characterize materials where the deflection of a high-frequency cantilever is used to measure the forces between the tip and the sample and map topography with nanoscale lateral resolution. As a result, AFM has been used to characterize the mechanical moduli of source rocks [2] and image the pores within kerogen [3]. The ability to map the mechanical moduli at the nanoscale and determine differences between the components is unique to AFM and not previously possible for lower resolution techniques. Distinguishing the organic and inorganic phases may explain how nanoscale properties influence the macroscale moduli measured from bulk rock. Such information is useful to explain differences in production in unconventional reservoirs.

The mechanical properties of the source rock, however, are only one set of information used to characterize unconventional reservoirs. Other parameters such as mineralogy and thermal maturity, which vary at the nanoscale in source rocks, are also important. The mineralogy provides information about the sediment source, and the thermal maturity and kerogen type determine the hydrocarbons generated. Kerogen is chemically complex and changes in crosslink density, porosity, molecular structure through maturation. Traditional AFM modes do not provide spectral information and other techniques are needed for chemical characterization. Fourier transform infrared (FTIR) spectroscopy provides a method to characterize organic matter from unconventional reservoirs $[1,4,5]$. FTIR detects spectra from a several-millimeter spot, micro-FTIR reduces the spot size to tens of microns, and with a focal plane array this region is reduced to several microns [1]. Optical detection is diffraction limited and does not provide sufficient resolution to study multicomponent materials with nanoscale heterogeneity. Additionally, multi-component averaging convolutes peaks in the fingerprint region leading to difficulties assigning these peaks in heterogeneous, multicomponent materials [1].

To meet this challenge a number of high resolution AFM-based spectral (i.e. Raman and FTIR) characterization methods have been developed where the detection is no longer optical but rather the forces acting on the tip at the end of a cantilever are used [6-8]. For example one AFM-IR method, previously demonstrated on shale, is photothermal IR (PTIR) which measures IR spectra in contact mode with a low-frequency cantilever at $\sim 100 \mathrm{~nm}$ resolution from thermal expansion [6, 9]. This method depends on differences in absorption and the coefficient of thermal expansion of the components, which may vary significantly depending upon the 3D-composition of rock. Typical acquisition time for an image at one laser wavelength is 25 minutes for PTIR. An alternative method is photo-induced force microscopy (PiFM) [7], where the AFM operates in non-contact mode and an induced dipole-dipole interaction is detected, which does not rely on thermal expansion. Rather, when the laser frequency matches the absorbance of the material a strong attractive force is detected as a change in the oscillation of the high-frequency cantilever. The FTIR spectrum is produced by varying 
the laser frequency at each pixel, and the AFM cantilever response is then converted to the relative IR absorbance. This process is repeated as the AFM tip is rastered above the surface to produce a highresolution hyperspectral IR map in a few hours, where the best lateral resolution is $<10 \mathrm{~nm}$.

Here we demonstrate the application of PiFM to provide nanoscale hyperspectral characterization of ion milled unconventional reservoir rock from the Barnett Formation using the Vista-IR (Molecular Vista, San Jose, CA). Figure 1 shows a representative $7 \mu \mathrm{m} \times 7 \mu \mathrm{m}$ topography (Fig. 1a) and hyperspectral (hyPIR) (Fig. 1b) image (256 x 256 pixels, $27 \times 27 \mathrm{~nm} /$ pixel, $\left.765-1899 \mathrm{~cm}^{-1} / \mathrm{pixel}\right)$. The topography alone (Fig. 1a) does not indicate obvious chemical heterogeneities; the black regions are pores. In contrast, the hyPIR image (Fig. 1b) provides a spectrum at each pixel and in gray scale shows significant nanoscale chemical variation. Figure 1c shows representative spectra from 10 positions along the line in Fig. 1b. The spectral peaks at each pixel in the hyPIR image can be used to categorize the sample and, for example, separate quartz (red band, Fig. 1c) and organic (green band, Fig. 1c) components into separate maps (Fig. 1d and 1e). In addition, variations in mineralogy are distinguishable and the organics might be sorted by either aromatic-rich or aliphatic-rich, a key indicator of the thermal maturity.

The data show that PiFM can map the nanoscale heterogeneities of unconventional reservoir rock. Because of the short-range dipole-dipole interaction, PiFM's lateral resolution is better than $10 \mathrm{~nm}$. At this resolution, the spectral peaks are those of the individual materials rather than a convolution of the peaks, enabling the characterization with the fingerprint region. In addition, the rapid response of the cantilever for PiFM detection provides a full hyperspectral dataset in just a few hours. A nanoscale understanding of the rock in unconventional reservoirs could have significant implications determining hydrocarbon potential and provide new insights for production [10].

\section{References:}

[1] Y Chen et al., International Journal of Molecular Sciences 16 (2015), p. 30223.

[2] M Eliyahu et al., Marine and Petroleum Geology 59 (2015), p. 294.

[3] F Javadapour, M Moravvej Farshi, and M Amrein, Journal of Canadian Petroleum Technology 51 (2012), p.236.

[4] M Vandenbroucke and C Largeau, Organic Geochemistry 38 (2007), p. 719.

[5] CH Sondergeld et al., SPE Unconventional Gas Conference (2010) SPE131768.

[6] A Dazzi and CB Prater, Chemical Reviews 117 (2016), p. 5146.

[7] D Nowak et al., Science Advances 2 (2016) p. e1501571.

[8] E Betzig et al., Science 251 (1991) p. 1468.

[9] J Yang et al., Nature Communications 8 (2017) p. 2179.

[10] We thank Katie Park, Jordan Kone, and Martin Poitzsch for data collection and research support.

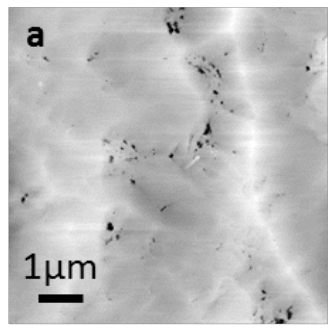

Topography

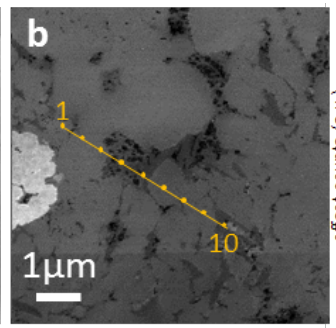

hyPIR

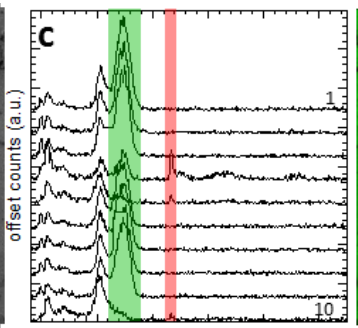

80010001200140016001800

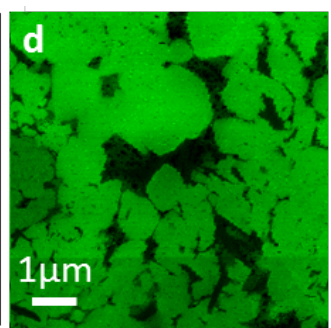

$1100 \mathrm{~cm}^{-1}$

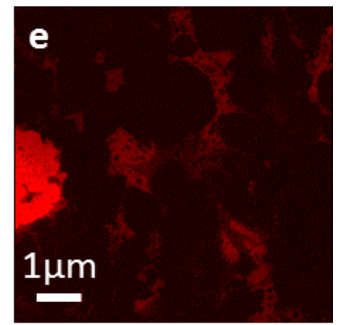

$1265 \mathrm{~cm}^{-1}$

Figure 1. Representative (a) topography, (b) hyPIR image, (c) spectra, (d) $1100 \mathrm{~cm}^{-1}$ map, and (e) $1265 \mathrm{~cm}^{-1}$ map. The hyPIR data shows significant chemical variations where the topography provides little information. The spectra can be used to distinguish quartz (green band) (d) from organics (red band) (e). 